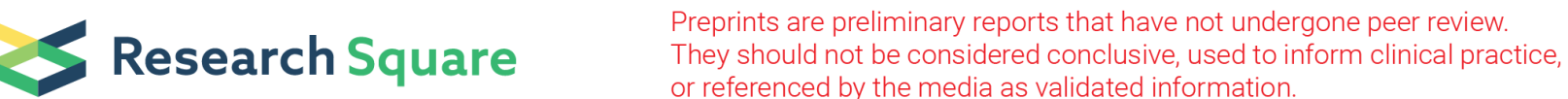

\section{Comparative Analysis of Pediatric Anxiety Measures in Clinical Sample: Evaluation of the PROMIS Pediatric Anxiety Short Forms}

John Parkhurst ( $\nabla$ jtparkhurst@luriechildrens.org )

Ann and Robert H Lurie Children's Hospital of Chicago https://orcid.org/0000-0002-6375-0883

Tara Von Mach

Ann and Robert $\mathrm{H}$ Lurie Children's Hospital of Chicago

Anthony T. Vesco

Ann and Robert H Lurie Children's Hospital of Chicago

Caroline E. Kerns

Ann and Robert H Lurie Children's Hospital of Chicago

John V. Lavigne

Ann and Robert H Lurie Children's Hospital of Chicago

\section{Research Article}

Keywords: Anxiety Disorder, PROMIS, Evidence-Based Assessment, Children

Posted Date: January 18th, 2022

DOI: https://doi.org/10.21203/rs.3.rs-1108724/v1

License: (c) (i) This work is licensed under a Creative Commons Attribution 4.0 International License. Read Full License 


\section{Abstract}

Objective: Examine the psychometric properties, validity in relation to a legacy measure, and diagnostic accuracy of the PROMIS Anxiety Short Form 2.0 (PROMIS A-SF) Caregiver and Youth Reports in a clinical sample.

Methods: Participants were 301 youth and caregivers referred to a behavioral health clinic by their pediatrician. Participants and their caregivers completed PROMIS A-SF (youth and caregiver proxy), SCARED (youth and caregiver proxy), and a semi-structured interview. Descriptive, correlational, psychometric, and receiver operating characteristic (ROC) analyses were conducted for both measures.

Results: PROMIS A-SF measures were highly correlated with SCARED total scores and the panic subscale. PROMIS A-SF measures had AUCs ranging from .49-.79 for the detection of any of three primary subtypes of anxiety: Generalized Anxiety, Separation Anxiety, and Social Anxiety.

Implications: Dimensional anxiety subtypes, such as Social Anxiety may not be well detected on the PROMIS youth measure. Use of the PROMIS A-SF as a part of Evidence Based Assessment process is discussed.

\section{Background}

Anxiety Disorders are the most common mental health concern in childhood and adolescence [1], resulting in significant disease burden for youth and their families [2]. Patient-report measures can play an important role in evidence-based assessment (EBA) for anxiety [3]. However, the use of patient-report measures vary due to challenges with measure selection (e.g. psychometrics, reading level, cost, length) and interpretation (e.g. score comparability to other measures) [4-6]. Further research investment is needed to help clinicians choose self-report measures that improve detection and diagnosis of pediatric anxiety in clinical samples.

While there are multiple self-report pediatric anxiety measures [7],the 41-item Screen for Child Anxiety and Related Emotional Disorder (SCARED) is among the most widely used "legacy" measures of pediatric anxiety $[8,10]$. The caregiver- and youth-report versions of the SCARED were developed using classic test theory. The SCARED has strong psychometric properties and is readily accessible (e.g. no cost and available in multiple languages). The SCARED is a dimensional measure of common anxiety disorders based on the Diagnostic and Statistical Manual-V (DSM-5) (American Psychiatric Association, 2018) [11] with subscales for Separation Anxiety (SEP), Generalized Anxiety (GAD), Social Anxiety (SOC), and Panic/Somatic symptoms, and School Avoidance. Cut off scores for each dimension are used to indicate the presence of a disorder [12].

The Patient Reported Outcomes Measurement Information System (PROMIS) was developed by the National Institute of Health $(\mathrm{NIH})$ to improve self-report measures. For each PROMIS measure, extensive item pools were developed by expert focus groups, and Item Response Theory (IRT) techniques were used 
to identify items invariant across race/ethnicity, sex, and age [13]. The youth and caregiver report PROMIS Pediatric Anxiety Short Forms 2.0 (PROMIS A-SF) are brief (e.g. 8 items), readily accessible, and result in a standardized score (i.e. T-Scores) $[14,15]$.

There are clear advantages to the PROMIS approach to test construction but also practical limitations. First, as Nunnally [16] noted, any test's validity needs to be assessed relative to (a) the specific construct(s) it is supposed to be measuring; (b) the application of the measure (e.g. screening, diagnosis, etc.); (c) the population with which it is to be used (age, clinical sample etc.). To do this, the classic psychometric properties of the PROMIS A-SF, including reliability and concurrent validity with "legacy" anxiety scales need to be examined as has been done with the PROMIS measures for pediatric depression [17]. Second, the PROMIS A-SF measure was developed as a unitary measure of anxiety that has not been assessed against specific anxiety disorders as defined by the DSM-V. As the authors of the PROMIS measures indicate, clinician-researchers are expected to further evaluate the strength of association between the measure and any disorder identified in clinical samples. For this reason, the relationship of the unitary construct of anxiety as measured by the PROMIS anxiety scale needs to be compared to each of the identified types of anxiety commonly seen in clinics and considered disorders in the DSM-5. Third, future directions for research on PROMIS measures include finding ways to implement their use in diverse clinical settings and identifying clinical thresholds for these measures [18]. Presently, little progress has been made in developing a research base to support the clinical applications of the PROMIS A-SF scale. For the PROMIS A-SF to be clinically useful, its diagnostic accuracy needs to be assessed and meaningful cutoff scores need to be defined.

\section{Study Aims}

Our primary study aim was to examine the concurrent validity of the PROMIS A-SF compared to the SCARED using an outpatient psychiatry sample. To accomplish this, the single-factor PROMIS A-SF was assessed against three major subtypes of anxiety (SEP, GAD, SOC) identified in the DSM-5 and the SCARED. Along with concurrent validity, the test-retest reliability of the PROMIS A-SF was examined.

While examining the correlations between the PROMIS A-SF and the SCARED help establish whether the two measures test the same construct, it is not sufficient to establish the utility of the PROMIS for determining whether a child or adolescent meets criteria for an anxiety disorder based on a structured diagnostic interview. However, the PROMIS A-SF may be a useful part of the EBA process as a screening measure to support statistical decision making. For that reason, the second aim of this study was to examine the diagnostic accuracy of the PROMIS A-SF in relation to an evidence-based semi-structured interview using Receiver Operating Characteristic (ROC) analysis.

\section{Method}

All study procedures and retrospective data collection were approved by the Institutional Review Board at Lurie Children's Hospital. 


\section{Participants}

Youth and caregivers $(N=301)$ were referred by primary care pediatricians for an outpatient psychiatric evaluation. Pediatricians making these referrals were part of a program called Mood, Anxiety, ADHD Collaborative Care (MAACC) [19] designed to train and expedite mental health care access in small- to medium-sized community pediatric practices. Patients and caregivers were referred by their pediatrician for evaluation between June 2018 and October 2020. Table 1 includes patient demographics. Youth (ages 7-18 years, $M=12.93 ; S D=3.02$ ) and their caregivers completed measures in English or Spanish. Thirteen caregivers used the Spanish Language PROMIS and SCARED measures; all other youth measures were completed in English. A smaller sample of youth and caregivers $(n=52)$ who had completed measures 2-3 weeks prior to the diagnostic interview were asked to repeat the PROMIS A-SF measure on the date of the diagnostic interview to assess test-retest reliability.

\section{Measures}

PROMIS Anxiety Short Forms 2.0 (PROMIS A-SF)

The PROMIS A-SF measures were created to assess anxiety for children ages 8-17 and caregiver proxy from ages 5-17 [14, 15]. Likert response total scores range from 8 to $40(1=$ "never" to $5=$ "almost always"). Summed raw scores and associated T-Scores $(M=50, S D=10)$ are provided on the Health Measures website (https://www.healthmeasures.net/search-view-measures?task=Search.search). There are no established clinical cut off scores for the PROMIS A-SF, although T-score severity levels of mildmoderate and moderate-severe have been described by Carle et al. [20] in a large sample.

Screen for Child Anxiety Related Emotional Disorders (SCARED)

The SCARED is a 41-item questionnaire designed to assess a variety of anxiety symptoms occurring over the prior three months, with parallel caregiver- and child-report versions [9]. The SCARED allows for calculation of a total anxiety score (0-82) and has a five-dimension structure, with subscale scores for panic/somatic anxiety, SEP, GAD, SOC, and school avoidance. The SCARED has demonstrated discriminant validity between anxious and non-anxious youth, strong internal consistency (coefficient a of approximately .90), and favorable psychometrics in treatment-seeking samples $[9,10,12,21,22]$.

Anxiety Disorders Interview Schedule for DSM-IV: Child and Parent Versions (ADIS-IV-C/P)

The ADIS-IV-C/P [23] are semi-structured diagnostic interviews used to assess psychopathology among youth ages 6-18, with a particular emphasis on anxiety disorders. Clinical Severity Ratings (CSR) ranging from 0 to 8 are assigned by the clinician for each diagnosis with the ADIS-IV-C/P, with a CSR of 4 or greater representing symptoms and distress/interference at a level that meets full diagnostic criteria. There is strong evidence supporting the reliability, validity, and sensitivity to clinical change for the ADISIV-C/P [24]. Test-retest reliability for anxiety disorder diagnoses for both parent and child reports is excellent ( $\nabla$ coefficients, .80 to .92 ) [24], and interrater agreement for anxiety disorders diagnosed with the

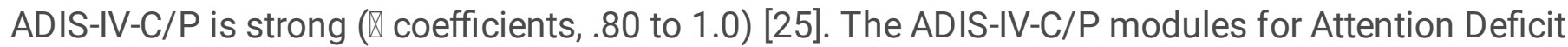


Hyperactivity Disorder, Oppositional Defiant Disorder, Separation Anxiety, Generalized Anxiety, Social Anxiety, Panic Disorder, and Depression were administered to all youth and caregivers.

\section{Procedure \& Data Collection}

Patients were referred to MAACC for further mental health evaluation by their pediatrician. Patients were initially screened by telephone for appropriateness of referral (e.g., age 7-18 years, no autism or developmental disorders, not recently engaged in higher levels of care, etc.). All patients and caregivers received the PROMIS A-SF and SCARED (among other clinical measures not included in this study) by mail or accessed online through a secure portal prior to their appointment. Directions were given to both the caregiver and youth to complete the measures prior to the diagnostic intake. The psychologist was occasionally presented with completed paper measures at intake if the patient did not complete measures digitally. This procedure represented the typical evidence based assessment process in a busy outpatient clinic described by Youngstrom and colleagues [26] and Ford-Paz et al. [27]. A licensed psychologist administered the ADIS-IV-C/P anxiety modules with the child or adolescent and at least one caregiver/guardian. Although inter-rater reliability was not tracked, the psychologist and program psychiatrist, both trained in ADIS-IV administration, discussed findings for all new diagnostic evaluations.

\section{Statistical Analysis}

Descriptive statistics were completed for the sample in addition to test-retest reliability with a subset ( $n=$ 52) participants who repeated measures. All analyses were conducted using STATA 15.1 [28].

Pearson's correlation coefficients were calculated to assess concurrent validity between the PROMIS A-SF and the SCARED (Total Score, Panic, GAD, Separation, Social Anxiety). Pearson correlations of $\geq .9$ are described as very high; .7-.89, high; .5-.69, moderate; .3-.49, low; <3, negligible [29].

Clinician-completed ADIS-IV structured interviews were administered to caregivers and youth and used to identify anxiety-related diagnoses of GAD, SEP, or SOC. The category of "any anxiety disorder" included children meeting criteria for any of those three anxiety disorders. ROC analyses were performed to determine how well the PROMIS and SCARED measures could distinguish between the presence or absence of each disorder. Panic disorder was not included because there were too few cases $(n=2)$ to allow for analyses. The Area Under the Curve (AUC) is the metric for the best overall classification ability for each diagnosis. AUC values are typically evaluated on the following scale: 0.9-1.0 excellent; 0.800.9.89 good; 0.70-0.79 fair; 0.60-0.69 poor; 0.50-0.59 fail. Sensitivity (SE), specificity (SP), and positive predictive values (PPV) were calculated for the PROMIS A-SF.

The percent of missing data was low for SCARED and PROMIS A-SF measures. Youth and Caregiver measures for SCARED-Total, Panic, GAD, SEP, SOC, and School Avoidance subscales had less than 13\% missing data. Missing data for the youth and caregiver measures of the PROMIS A-SF Total Scores was less than $6 \%$. To determine if the data were Missing Completely at Random (MCAR), Little's test was 
performed and was not found to be significant $(p=.57)$. As a result, imputation was not needed and pairwise deletion was used to manage missing data.

\section{Results}

\section{Descriptive Statistics}

Key demographic descriptive statistics are reported in Table 1. The average age of patients was 12.93 years $(S D=3.02)$. Approximately $70.43 \%(n=212)$ of the sample was White and $16.94 \%$ was Hispanic.

On the ADIS-IV, 18 (5.98\%) patients met criteria for SEP; 111 (36.88\%) met criteria GAD; 47 (15.61\%) met criteria for SOC; and 2 (.66\%) met criteria for Panic Disorder. Anxiety Disorder Not Otherwise Specified (Anxiety Disorder NOS) was identified in 58 (19.27\%) patients, indicating the presence of anxiety symptoms without significant functional impact. One or more comorbid diagnoses were observed in $67.44 \%$ of patients $(n=203)$.

Paired sample $t$-tests of youth and caregiver PROMIS A-SF total score means and the youth and caregiver SCARED total score means did not significantly differ by race, ethnicity, and insurance type. However, mean scores were significantly higher in females compared to males on the youth PROMIS A-SF $(t=$ $-4.69 ; p<0.001)$, caregiver PROMIS A-SF $(t=-2.16 ; p<0.05)$, youth SCARED total score $(t=-4.40 ; p<$ $0.001)$, and the caregiver SCARED total score $(t=-2.49 ; p<0.05)$.

The test re-test correlation coefficient as measured by Pearson's correlation coefficient on the youth PROMIS A-SF was $r=0.75(p<.001 ; n=41)$ and the caregiver PROMIS A-SF was $r=0.73(p<0.001 ; n=$ 38).

\section{Concurrent Validity}

Table 2 includes the correlations between the PROMIS A-SF and the anxiety subscales of the SCARED. For the youth measures, the PROMIS A-SF correlated moderately highly with the SCARED total $(r=0.697$; $p<0.001), \operatorname{GAD}(r=0.596 ; p<0.001)$, and Panic scale $(r=0.665 ; p<0.001)$. The youth PROMIS A-SF had a low correlation with the Youth SCARED SEP $(r=0.437 ; p<0.001)$ and a negligible correlation with SCARED SOC $(r=0.279 ; p<0.001)$. Differences in the magnitude of these correlations were examined using Fisher's $z$-transformation. The correlations between the PROMIS A-SF and both the SCARED SEP ( $z$ $=4.80, p<.001)$ and SCARED SOC $(z=4.80, p<.001)$ were significantly lower than the correlation between the PROMIS A-SF scale and the SCARED total score.

The caregiver proxy PROMIS A-SF measure was moderately highly correlated with the caregiver SCARED Total $(r=0.684 ; p<0.001)$ and caregiver SCARED SEP $(r=0.640 ; p<0.001)$, the SCARED GAD $(r=0.565$; $p<0.001)$, and the SCARED Panic scale $(r=0.599 ; p<0.001)$. The correlation of the caregiver proxy PROMIS A-SF and the SCARED SOC was negligible $(r=0.214 ; p<0.001)$. The correlation between the PROMIS anxiety scale and SCARED social anxiety scale $(z=4.80, p<.001)$ was significantly lower than the correlation between the PROMIS anxiety scale and the SCARED total anxiety score. 


\section{Diagnostic Accuracy}

Table 3 presents a comparison of AUC values for Caregiver and Youth PROMIS A-SF Total score, SCARED

Total score and SCARED subscales (SOC, GAD, SEP) in accurately diagnosing Separation Anxiety,

Generalized anxiety, Social Anxiety, or any anxiety disorder (e.g. GAD, SEP, or SOC).

The AUC obtained for the Youth PROMIS A-SF considered fairly good for identifying any anxiety disorder was $0.72(95 \% \mathrm{Cl}=0.66-0.78)$; fair for GAD, $0.73(95 \% \mathrm{Cl}=0.67-0.79)$; and fair for SEP, $0.71(95 \% \mathrm{Cl}=$ 0.57-0.85). Youth PROMIS A-SF AUC for SOC was poor and did not exceed chance $(0.49,95 \% \mathrm{Cl}=0.40-$ 0.58). The AUC obtained for Caregiver PROMIS A-SF was fair for any anxiety disorder $(0.74,95 \% \mathrm{Cl}=$ 0.64-0.76), GAD (0.71, 95\% $\mathrm{Cl}=0.65-0.77)$ and SEP $(0.79,95 \% \mathrm{Cl} 0.63-0.94)$. Caregiver PROMIS A-SF AUC for SOC was poor $(0.54,95 \% \mathrm{Cl}=0.45-0.62)$.

Caregiver and Youth SCARED Total Score and SCARED Subscales AUC values ranged from fair (0.72) to excellent (0.91).

SE, SP, PPVs, and associated clinical cut offs and T-scores are presented in Table 3 for the PROMIS and SCARED measures. For a screening measure an ideal cutoff score generates an SE of $\geq .80$ and an SP of 2.80. None of the PROMIS measures generated a score meeting those SE and SP values. In our sample, this standard was only approached for the caregiver PROMIS A-SF SEP (.82; .72.).

\section{Discussion}

This study examined the concurrent validity of the youth and caregiver proxy PROMIS A-SF by comparing it with a legacy pediatric anxiety measure, the SCARED, and assessed the diagnostic accuracy of the PROMIS A-SF measures as predictors of the three major subtypes of anxiety disorders derived from an evidence-based structured diagnostic interview. Using the ADIS-IV-C/P, 18 (5.98\%) patients met criteria for SEP; 111 (36.88\%) met criteria GAD; 47 (15.61\%) met criteria for SOC. One or more comorbid diagnoses were observed in $67.44 \%$ of patients $(n=203)$.

Assessing the concurrent validity of a new measure (e.g., PROMIS A-SF) in comparison to a legacy measure (e.g. SCARED), both ostensibly measuring the same construct, helps establish whether the underlying construct is indeed the same for the two measures. Because the goal of developing the PROMIS measures was to produce caregiver- and youth-report anxiety scales with a single factor structure invariant across age, sex, and race/ethnicity, it was likely that the PROMIS A-SF would not do equally well in assessing the several different dimensions of anxiety assessed on the SCARED [9], [10]. As a result, it is not surprising that significant differences were detected in the magnitude of the correlations between the PROMIS A-SF and the SCARED subscales for both youth- and caregiver-report. The results indicate that the caregiver PROMIS A-SF provides a better estimate of the underlying construct for total anxiety, panic, and generalized anxiety, whereas the youth PROMIS A-SF demonstrated strong relationships with total anxiety and panic. The correlation of the youth PROMIS A-SF measure was 
significantly lower for the SEP and SOC subscales. The caregiver PROMIS A-SF performed adequately with SEP (.640).

This pattern of results suggest that (a) if a researcher or clinician is seeking a brief, caregiver-reported anxiety scale measuring the same underlying constructs as the SCARED total anxiety, SEP, GAD, or panic scales, the caregiver report PROMIS A-SF is a reasonable choice; (b) if the researcher or clinician is seeking a brief caregiver report measure of SOC as measured by the SCARED, the PROMIS A-SF would not be appropriate; (c) similarly, if the researcher is seeking a brief youth report measuring the same constructs as the SCARED total, GAD, and panic scales, the PROMIS A-SF is a reasonable choice, but the measure would be less appropriate for measuring SOC or SEP.

Understanding the role that the PROMIS A-SF might play in EBA has received little attention to date and could have practical implications for improving EBA procedures with children, adolescents, and their caregivers. NIH priorities for future research on PROMIS measures include identifying thresholds to improve the clinical applications of these measures. Finding ways to implement EBA techniques in busy clinical settings and optimize their utility may well involve applications of precision mental health procedures in the future [30]. In a precision mental health approach, the likelihood that an individual will meet criteria for a disorder or will respond to treatment is calculated and can be used for clinical decisionmaking.

Currently the PROMIS measures may be incorporated into recommended EBA practices. For example, Youngstrom and Van Meter [22] have described the use of nomograms to improve statistical prediction of diagnosis in in clinic settings using the identified prevalence of a disorder in a local sample and brief screening tools with known SE and SP, such as the PROMIS A-SF. Considering the present sample, the prevalence of GAD based on semi-structured interview was $36.88 \%$ and the calculated accuracy of the youth PROMIS A-SF in detection of GAD was SE $=69.16$; SP $=68.16$ (T-Score 58.7). An above cut off score would result in an increased probability that a youth would meet criteria for a diagnosis of GAD (56\%), whereas below cut off score would decrease the probability $(21 \%)$ of a GAD diagnosis. In this example, a measure being above or below the cut off significantly improves prediction of a disorder's presence or absence over the starting point of population prevalence. While "optimal" (SE = .8, SP = .8) cut off scores were not achieved by any of the PROMIS A-SF in our sample, knowledge of local prevalence of a disorder of interest and SE and SP a measure of that clinical condition can improve the process of statistical prediction resulting in better accuracy with diagnosis. Additionally, while structured interviews remain the gold standard for diagnostic decision-making, they are underutilized in clinic settings because of their length and/or administration time [31]; instruments like the PROMIS $₫$ measures might be useful in determining which modules of a structured interview should or should not be administered to shorten administration time of a structured interview [27].

The SCARED and PROMIS A-SF are measuring the broad construct of total anxiety and both measures can be clinical valuable in EBA practice. Both caregiver and youth PROMIS A-SF measures the presence of any anxiety reasonably well. Additionally, test-retest reliability of the PROMIS A-SF measure was 
strong. Thus, the PROMIS A-SF may be particularly useful as an initial screener for any anxiety symptoms or for progress monitoring during treatment.

\section{Strength and Limitations}

The strengths of this study include the novel investigation of the PROMIS A-SF compared to a gold standard semi-structured interview (ADIS-IV-C/P) in an outpatient clinical psychiatry sample. The sample of 301 youth and caregivers is well powered to draw assumptions of the performance of the PROMIS ASF and missing data was minimized $(<13 \%)$. This study is the first to describe the relationship between the youth and caregiver PROMIS A-SF report with a legacy anxiety measure and report psychometric properties of the PROMIS A-SF in a clinical sample. Findings suggest consideration for use of the PROMIS A-SF in clinical practice. Interestingly, this study provides the first test-retest reliability data for the PROMIS A-SF, which is valuable in assessing reliable-change, if the PROMIS A-SF is used as a progress monitoring tool.

The ADIS-IV-C/P anxiety modules were completed by a trained evaluator with the youth and caregiver. The evaluator was not blinded to SCARED or PROMIS measures but did not have scoring of these measures available prior to the interview. As has been discussed [27], while non-blinding the evaluator may introduce bias, it also reflects real world clinical practice. Measures of inter-rater reliability for the ADIS-IV was not collected but interviews were completed by a trained psychologist and reviewed by a trained psychiatrist.

\section{Conclusion}

The PROMIS A-SF are brief, psychometrically sound, instruments created for the purpose of assessing symptomology across a range of health conditions. The SCARED is a widely used, well-established measure of anxiety. Youth and caregiver PROMIS A-SF results are comparable to the SCARED in the detection of any anxiety disorder, however, appear limited in the detection of specific subtypes of anxiety including social anxiety and separation anxiety in a general psychiatry sample. Specific consideration of anxiety disorders of interest should be taken when choosing how to best incorporate the PROMIS A-SF into your research and clinical practice.

\section{Declarations}

The authors have no conflicts of interest to disclose.

We would like to acknowledge Lurie Children's Hospital Healthy Communities for providing initial funding and the Pritzker Foundation for ongoing funding for Lurie Children's Hospital's collaborative care program.

\section{References}


1. Merikangas, K. R., He, J.-P., Burstein, M., Swanson, S. A., Avenevoli, S., Cui, L., Benjet, C., Georgiades, K., \& Swendsen, J. (2010). Lifetime prevalence of mental disorders in U.S. adolescents: Results from the National Comorbidity Survey Replication-Adolescent Supplement (NCS-A). Journal of the American Academy of Child and Adolescent Psychiatry, 49(10), 980-989.

https://doi.org/10.1016/j.jaac.2010.05.017

2. Bushnell, G. A., Gaynes, B. N., Compton, S. N., Dusetzina, S. B., Brookhart, M. A., \& Stürmer, T. (2019). Incidence of mental health hospitalizations, treated self-harm, and emergency room visits following new anxiety disorder diagnoses in privately insured U.S. children. Depression and Anxiety, 36(2), 179-189. https://doi.org/10.1002/da.22849

3. Jensen-Doss, A. (2015). Practical, evidence-based clinical decision making: Introduction to the special series. Cognitive and Behavioral Practice, 22(1), 1-4. https://doi.org/10.1016/j.cbpra.2014.08.001

4. Chekroud, A. M., Zotti, R. J., Shehzad, Z., Gueorguieva, R., Johnson, M. K., Trivedi, M. H., Cannon, T. D., Krystal, J. H., \& Corlett, P. R. (2016). Cross-trial prediction of treatment outcome in depression: A machine learning approach. The Lancet. Psychiatry, 3(3), 243-250. https://doi.org/10.1016/S22150366(15)00471-X

5. McHugh, R. K., Rasmussen, J. L., \& Otto, M. W. (2011). Comprehension of self-report evidence-based measures of anxiety. Depression and Anxiety, 28(7), 607-614. https://doi.org/10.1002/da.20827

6. Schalet, B. D., Cook, K. F., Choi, S. W., \& Cella, D. (2014). Establishing a Common Metric for SelfReported Anxiety: Linking the MASQ, PANAS, and GAD-7 to PROMIS Anxiety. Journal of Anxiety Disorders, 28(1), 88-96. https://doi.org/10.1016/j.janxdis.2013.11.006

7. Wehry, A. M., Beesdo-Baum, K., Hennelly, M. M., Connolly, S. D., \& Strawn, J. R. (2015). Assessment and Treatment of Anxiety Disorders in Children and Adolescents. Current Psychiatry Reports, 17(7), 591. https://doi.org/10.1007/s11920-015-0591-z

8. Behrens, B., Swetlitz, C., Pine, D. S., \& Pagliaccio, D. (2019). The Screen for Child Anxiety Related Emotional Disorders (SCARED): Informant discrepancy, measurement invariance, and test-retest reliability. Child Psychiatry and Human Development, 50(3), 473-482. https://doi.org/10.1007/s10578-018-0854-0

9. Birmaher, B., Brent, D. A., Chiappetta, L., Bridge, J., Monga, S., \& Baugher, M. (1999). Psychometric Properties of the Screen for Child Anxiety Related Emotional Disorders (SCARED): A Replication Study. Journal of the American Academy of Child \& Adolescent Psychiatry, 38(10), 1230-1236. https://doi.org/10.1097/00004583-199910000-00011

10. Birmaher, B., Khetarpal, S., Brent, D., Cully, M., Balach, L., Kaufman, J., \& Neer, S. M. (1997). The Screen for Child Anxiety Related Emotional Disorders (SCARED): Scale construction and psychometric characteristics. Journal of the American Academy of Child and Adolescent Psychiatry, 36(4), 545-553. https://doi.org/10.1097/00004583-199704000-00018

11. American Psychiatric Association. (2013). Diagnostic and Statistical Manual of Mental Disorders (Fifth Edition). American Psychiatric Association. 
https://doi.org/10.1176/appi.books.9780890425596

12. Rappaport, B. I., Pagliaccio, D., Pine, D. S., Klein, D. N., \& Jarcho, J. M. (2017). Discriminant validity, diagnostic utility, and parent-child agreement on the Screen for Child Anxiety Related Emotional Disorders (SCARED) in treatment- and non-treatment-seeking youth. Journal of Anxiety Disorders, 51, 22-31. https://doi.org/10.1016/j.janxdis.2017.08.006

13. Bevans, K. B., Gardner, W., Pajer, K. A., Becker, B., Carle, A., Tucker, C. A., \& Forrest, C. B. (2018). Psychometric Evaluation of the PROMIS ${ }^{\circledR}$ Pediatric Psychological and Physical Stress Experiences Measures. Journal of Pediatric Psychology, 43(6), 678-692. https://doi.org/10.1093/jpepsy/jsy010

14. Irwin, D. E., Gross, H. E., Stucky, B. D., Thissen, D., DeWitt, E. M., Lai, J. S., Amtmann, D., Khastou, L., Varni, J. W., \& DeWalt, D. A. (2012). Development of six PROMIS pediatrics proxy-report item banks. Health and Quality of Life Outcomes, 10, 22. https://doi.org/10.1186/1477-7525-10-22

15. Varni, J. W., Magnus, B., Stucky, B. D., Liu, Y., Quinn, H., Thissen, D., Gross, H. E., Huang, I.-C., \& DeWalt, D. A. (2014). Psychometric properties of the PROMIS $®$ pediatric scales: Precision, stability, and comparison of different scoring and administration options. Quality of Life Research: An International Journal of Quality of Life Aspects of Treatment, Care and Rehabilitation, 23(4), 12331243. https://doi.org/10.1007/s11136-013-0544-0

16. Nunnally, J. C. (1955). A systematic approach to the construction of hypotheses about the process of psychotherapy. Journal of Consulting Psychology, 19(1), 17-20. https://doi.org/10.1037/h0046840

17. Clover, K., Lambert, S. D., Oldmeadow, C., Britton, B., King, M. T., Mitchell, A. J., \& Carter, G. (2018). PROMIS depression measures perform similarly to legacy measures relative to a structured diagnostic interview for depression in cancer patients. Quality of Life Research, 27(5), 1357-1367. https://doi.org/10.1007/s11136-018-1803-x

18. Smith, A. W., \& Jensen, R. E. (2019). Beyond methods to applied research: Realizing the vision of PROMIS ${ }^{\circledR}$. Health Psychology, 38(5), 347-350. https://doi.org/10.1037/hea0000752

19. Parkhurst, J. T., Ballard, R. R., Lavigne, J. V., Von Mach, T., Romba, C., Perez-Reisler, M., \& Walkup, J. T. (2021). Extending collaborative care to independent primary care practices: A chronic care model. Clinical Practice in Pediatric Psychology. https://doi.org/10.1037/cpp0000383

20. Carle, A. C., Bevans, K. B., Tucker, C. A., \& Forrest, C. B. (2020). Using nationally representative percentiles to interpret PROMIS pediatric measures. Quality of Life Research. https://doi.org/10.1007/s11136-020-02700-5

21. Monga, S., Birmaher, B., Chiappetta, L., Brent, D., Kaufman, J., Bridge, J., \& Cully, M. (2000). Screen for child anxiety-related emotional disorders (SCARED): Convergent and divergent validity. Depression and Anxiety, 12(2), 85-91. https://doi.org/10.1002/1520-6394

22. Youngstrom, E. A., \& Meter, A. V. (2016). Empirically Supported Assessment of Children and Adolescents. Clinical Psychology: Science and Practice, 23(4), 327-347.

https://doi.org/10.1111/cpsp.12172

23. Silverman, W. K., \& Ollendick, T. H. (2005). Evidence-based assessment of anxiety and its disorders in children and adolescents. Journal of Clinical Child and Adolescent Psychology: The Official Journal 
for the Society of Clinical Child and Adolescent Psychology, American Psychological Association, Division 53, 34(3), 380-411. https://doi.org/10.1207/s15374424jccp3403_2

24. Silverman, W. K., Saavedra, L. M., \& Pina, A. A. (2001). Test-Retest Reliability of Anxiety Symptoms and Diagnoses With the Anxiety Disorders Interview Schedule for DSM-IV: Child and Parent Versions. Journal of the American Academy of Child \& Adolescent Psychiatry, 40(8), 937-944. https://doi.org/10.1097/00004583-200108000-00016

25. Lyneham, H. J., Abbott, M. J., \& Rapee, R. M. (2007). Interrater reliability of the Anxiety Disorders Interview Schedule for DSM-IV: Child and parent version. Journal of the American Academy of Child and Adolescent Psychiatry, 46(6), 731-736. https://doi.org/10.1097/chi.0b013e3180465a09

26. Youngstrom, E. A., Choukas-Bradley, S., Calhoun, C. D., \& Jensen-Doss, A. (2015). Clinical guide to the evidence-based assessment approach to diagnosis and treatment. Cognitive and Behavioral Practice, 22(1), 20-35. https://doi.org/10.1016/j.cbpra.2013.12.005

27. Ford-Paz, R. E., Gouze, K. R., Kerns, C. E., Ballard, R., Parkhurst, J. T., Jha, P., \& Lavigne, J. (2020). Evidence-based assessment in clinical settings: Reducing assessment burden for a structured measure of child and adolescent anxiety. Psychological Services, 17(3), 343-354. https://doi.org/10.1037/ser0000367

28. STATA (2017). Statistical Software: Release 15. College Station, TX: StataCorp LLC.

29. Mukaka, M. (2012). A guide to appropriate use of Correlation coefficient in medical research. Malawi Medical Journal: The Journal of Medical Association of Malawi, 24(3), 69-71.

30. Bickman, L., Lyon, A. R., \& Wolpert, M. (2016). Achieving Precision Mental Health through Effective Assessment, Monitoring, and Feedback Processes. Administration and Policy in Mental Health, 43, 271-276. https://doi.org/10.1007/s10488-016-0718-5

31. Galanter, C. A., \& Patel, V. L. (2005). Medical decision making: A selective review for child psychiatrists and psychologists. Journal of Child Psychology and Psychiatry, 46(7), 675-689. https://doi.org/10.1111/j.1469-7610.2005.01452.x

\section{Tables}

Table 1. Sample Characteristics 


\begin{tabular}{|c|c|}
\hline Characteristic & Overall $(n=301)$ \\
\hline & $M(S D)$ or $n(\%)$ \\
\hline Age & $12.93(3.02)$ \\
\hline \multicolumn{2}{|l|}{ Gender } \\
\hline Male & $125(41.53 \%)$ \\
\hline Female & $176(58.47 \%)$ \\
\hline \multicolumn{2}{|l|}{ Ethnicity } \\
\hline Hispanic & $51(16.94 \%)$ \\
\hline Non-Hispanic & $232(77.08 \%)$ \\
\hline Declined or Unknown & $18(5.98 \%)$ \\
\hline \multicolumn{2}{|l|}{ Race } \\
\hline White & $212(70.43 \%)$ \\
\hline Black/African American & $12(3.99 \%)$ \\
\hline Asian & $18(5.98 \%)$ \\
\hline Biracial, Other & $50(16.61 \%)$ \\
\hline Public Insurance & $59(19.60 \%)$ \\
\hline Any Anxiety Diagnosis ${ }^{a}$ & $153(50.83)$ \\
\hline Separation Anxiety & $18(5.98 \%)$ \\
\hline GAD Diagnosis & $111(36.88 \%)$ \\
\hline Social Anxiety & $47(15.61 \%)$ \\
\hline Panic Disorder & $2(0.66 \%)$ \\
\hline Anxiety Disorder (NOS) & $58(19.27 \%)$ \\
\hline OCD b & $12(3.98 \%)$ \\
\hline Depression Diagnosis $^{c}$ & $99(32.89 \%)$ \\
\hline ADHD Diagnosis ${ }^{d}$ & $123(40.86 \%)$ \\
\hline ODD Diagnosis ${ }^{\mathrm{e}}$ & $28(9.30 \%)$ \\
\hline Trauma Diagnosis ${ }^{f}$ & $17(5.65 \%)$ \\
\hline Co-morbid diagnosis (1 or more diagnosis) & $203(67.44 \%)$ \\
\hline
\end{tabular}


Note. $a$ = Includes the following primary, secondary, tertiary, and quaternary diagnoses: GAD, Separation Anxiety, Social Anxiety, or Panic Disorder; $b=$ Includes the following primary, secondary, tertiary, and quaternary diagnoses: $\mathrm{OCD} ; \mathrm{c}=$ Includes the following primary, secondary, tertiary, and quaternary diagnoses: Depression (NOS), Major Depressive Disorder, Mood Disorder (NOS), Major Depressive Episode, Mood Disorder, Disruptive Mood Dysregulation Disorder; $d$ = Includes the following primary, secondary, tertiary, and quaternary diagnoses: ADHD-Inattentive Type, ADHD-Hyperactive Type, ADHDCombined Type; e = Includes the following primary, secondary, tertiary, and quaternary diagnoses: Disruptive Disorder, Conduct Disorder, Oppositional Defiant Disorder, Impulse Control Disorder; $f=$ Includes the following primary, secondary, tertiary, and quaternary diagnoses: PTSD, Trauma, Adjustment Disorder, Bereavement, Other Trauma

Table 2. Correlations PROMIS anxiety and SCARED anxiety scales for Youth and Caregiver Forms

\begin{tabular}{|ll|}
\hline & Youth PROMIS Anxiety SF \\
\hline 1. Youth SCARED Total & $0.697 \star \star \star$ \\
\hline 2. Panic & $0.665^{\star \star \star}$ \\
\hline 3. Generalized Anxiety & $0.596 \star \star \star$ \\
\hline 4. Separation & $0.437 \star \star \star$ \\
\hline 5. Social Anxiety & $0.279 \star \star \star$ \\
\hline & Caregiver PROMIS Anxiety SF \\
\hline 6. Caregiver SCARED Total & $0.684 \star \star \star$ \\
\hline 7. Panic & $0.599 \star \star \star$ \\
\hline 8. Generalized Anxiety & $0.565 \star \star \star$ \\
\hline 9. Separation & $0.640 \star \star \star$ \\
\hline 10. Social Anxiety & $0.214 \star \star \star$ \\
\hline
\end{tabular}

Note. ${ }^{\star} \mathrm{p}<0.05 ;{ }^{* \star} \mathrm{p}<0.01 ;{ }^{* \star *} \mathrm{p}<0.001 ;$ Mean (SD)

Table 3. Comparison of Area Under the Curve and cutoff scores for the PROMIS and SCARED 


\begin{tabular}{|c|c|c|c|c|c|c|c|c|}
\hline Scale & Diagnosis & $\mathrm{N}$ & AUC & $\begin{array}{l}\text { T- } \\
\text { Score }\end{array}$ & Cutoff & Sensitivity & Specificity & $\begin{array}{l}\text { Positive } \\
\text { Likelihood } \\
\text { Ratio }\end{array}$ \\
\hline \multirow[t]{4}{*}{$\begin{array}{l}\text { Youth } \\
\text { PROMIS }\end{array}$} & $\begin{array}{l}\text { Any Anxiety } \\
\text { Disorder* }\end{array}$ & 286 & 0.72 & 57.5 & $\geq 21$ & $65.99 \%$ & $65.47 \%$ & 1.91 \\
\hline & GAD & 286 & 0.73 & 58.7 & $\geq 22$ & $69.16 \%$ & $68.16 \%$ & 2.17 \\
\hline & $\begin{array}{l}\text { Social } \\
\text { Anxiety }\end{array}$ & 286 & 0.49 & 48.3 & $\geq 14$ & $71.74 \%$ & $22.08 \%$ & 0.92 \\
\hline & $\begin{array}{l}\text { Separation } \\
\text { Anxiety } \\
\text { (SAD) }\end{array}$ & 286 & 0.71 & 65.7 & $\geq 28$ & $56.25 \%$ & $81.11 \%$ & 2.98 \\
\hline \multirow{4}{*}{$\begin{array}{l}\text { Caregiver } \\
\text { PROMIS }\end{array}$} & $\begin{array}{l}\text { Any Anxiety } \\
\text { Disorder* }\end{array}$ & 294 & 0.74 & 54.7 & $\geq 17$ & $62.84 \%$ & $60.96 \%$ & 1.91 \\
\hline & GAD & 294 & 0.71 & 53.3 & $\geq 16$ & $69.44 \%$ & $57.53 \%$ & 1.64 \\
\hline & $\begin{array}{l}\text { Social } \\
\text { Anxiety }\end{array}$ & 294 & 0.54 & 39.7 & $\geq 9$ & $100.00 \%$ & $12.50 \%$ & 1.14 \\
\hline & $\begin{array}{l}\text { Separation } \\
\text { Anxiety } \\
\text { (SAD) }\end{array}$ & 294 & 0.79 & 60.1 & $\geq 21$ & $82.35 \%$ & $72.92 \%$ & 3.04 \\
\hline \multirow{5}{*}{$\begin{array}{l}\text { Youth } \\
\text { SCARED }\end{array}$} & $\begin{array}{l}\text { Any Anxiety } \\
\text { Disorder* }\end{array}$ & 265 & 0.77 & - & & $76.26 \%$ & $70.63 \%$ & 2.60 \\
\hline & & & & & $\geq 27$ & & & \\
\hline & GAD & 265 & 0.77 & - & $\geq 8$ & $86.27 \%$ & $53.37 \%$ & 1.85 \\
\hline & $\begin{array}{l}\text { Social } \\
\text { Anxiety }\end{array}$ & 265 & 0.72 & - & $\geq 6$ & $84.44 \%$ & $46.82 \%$ & 1.59 \\
\hline & $\begin{array}{l}\text { Separation } \\
\text { Anxiety } \\
\text { (SAD) }\end{array}$ & 265 & 0.88 & - & $\geq 6$ & $92.86 \%$ & $71.31 \%$ & 3.23 \\
\hline \multirow[t]{4}{*}{$\begin{array}{l}\text { Caregiver } \\
\text { SCARED }\end{array}$} & $\begin{array}{l}\text { Any Anxiety } \\
\text { Disorder* }\end{array}$ & 283 & 0.75 & - & $\geq 18$ & $74.31 \%$ & $59.71 \%$ & 1.84 \\
\hline & GAD & 283 & 0.72 & - & $\geq 7$ & $78.30 \%$ & $55.93 \%$ & 1.78 \\
\hline & $\begin{array}{l}\text { Social } \\
\text { Anxiety }\end{array}$ & 283 & 0.82 & - & $\geq 7$ & $77.27 \%$ & $72.38 \%$ & 2.8 \\
\hline & $\begin{array}{l}\text { Separation } \\
\text { Anxiety } \\
\text { (SAD) }\end{array}$ & 283 & 0.91 & - & $\geq 6$ & $81.25 \%$ & $83.90 \%$ & 5.05 \\
\hline
\end{tabular}

Note: * Any anxiety disorder includes Separation Anxiety Disorder, GAD, Social Anxiety Disorder, \& Panic Disorder; Total scores on the SCARED are compared to Any Anxiety Disorder (SEP, GAD, Social Anxiety, \& 
Panic Disorder); GAD, Social Anxiety Disorder, and Separation Anxiety Disorder are all compared to SCARED subscales that correspond to each diagnosis 